\title{
ESPAÑA-2015, EN EL CONTEXTO DE LA SALIDA DE LA RECESIÓN
}

\author{
Autores: M. Alejandro Cardenete ${ }^{I}$ \\ Catedrático de Economía \\ M. Carmen Delgado \\ Profesora Auxiliar \\ Universidad Loyola Andalucía
}

\section{Resumen}

En este trabajo se estudia el contexto en el que se encuentra España ante la inminente salida de la recesión económica. Para ello se ha llevado a cabo un análisis de la estructura de la economía española, con el fin de analizar los posibles efectos que la crisis económica ha tenido sobre la estructura productiva del país. Para ello, se emplea la información proporcionada por las Matrices de Contabilidad Social (MCS). Las Matrices de Contabilidad Social describen el flujo circular de la renta en una economía y aportan la base estadística necesaria para el análisis e iden-

\footnotetext{
${ }^{1}$ macardenete@uloyola.es

${ }^{2}$ mcdelgado@uloyola.es
} 


\title{
M. Alejandro Cardenete y M. Carmen Delgado
}

tificación de los sectores más importantes de la economía, denominados sectores clave. La metodología aplicada será un modelo MCS lineal a través del análisis de multiplicadores, identificando así los sectores productivos más relevantes de España. Por último, y con iniciativa de conocer cuál será el cierre del año 2015 y qué esperamos para el año 2016 en España, se han analizado las previsiones económicas de dos centros de estudios diferentes, comprobando que la salida de la crisis y el comienzo de un periodo de crecimiento económico parece ser una realidad.

Palabras clave: análisis económico; matrices de contabilidad social; proyecciones económicas.

\section{Spain-2015 in the context of the out of recession}

\begin{abstract}
This paper describes the context in which Spain is out of recession. For this, we have carried out an analysis of the structure of the Spanish economy, in order to analyse the effects of the economic crisis on the Spanish productive structure. To do this, the information provided by the Social Accounting Matrix (SAM) is used. Social Accounting Matrices describe the circular flow of income in an economy and provide the statistical basis for the analysis and identification of the most important sectors of the economy, called key sectors. The methodology will be a linear model MCS through multiplier analysis, identifying the most important productive sectors in Spain. Finally, as an initiative to know what happened with the economic growth at the end of 2015 and for 2016 in Spain, we have analysed the economic forecasts in two different works, ensuring both of them, the exit from the crisis and the beginning of a period of economic growth.
\end{abstract}

Key words: economic analysis; social sccounting matrices; economic forecast.

Recibido: 16-11-2015

Aceptado: 09-12-2015

\section{INTRODUCCIÓN}

A finales del pasado año ya todo parecía indicar que el año 2015 sería para España el año de la recuperación económica. Al comenzar el año, en el primer 
trimestre del mismo, se pudo observar un cambio de tendencia mostrado por algunas variables económicas, determinado por varios factores especialmente relevantes, entre los que se encontraban: la contención de los precios de los productos primarios y, en particular, el descenso progresivo del precio del petróleo desde junio del 2014; la fortaleza mostrada por el dólar con respecto a otras monedas, en especial frente al euro desde mediados de 2014; los tipos de interés moderados en el contexto internacional; la inestabilidad de los mercados financieros, aunque ahora menos acusada en la Unión Europea y Estados Unidos que durante la crisis; un comercio mundial que continúa sin despegar o la intensificación de algunas tensiones geopolíticas, que complican la gobernanza económica internacional para hacer frente a los retos derivados de una economía cada vez más polarizada, donde la rivalidad entre grandes potencias se está haciendo más patente.

Otra variable a tener en cuenta son las exportaciones netas. España concluyó el 2014 alcanzando un máximo histórico de exportaciones (240.034,9 millones de euros). Este comportamiento favorable se vio neutralizado, en cierta media, por el fuerte impulso de las importaciones (que aumentaron el doble que las exportaciones) y, de manera simultánea, por la recuperación de la demanda interna, lo cual ha determinado que el déficit comercial español creciese un 53,4\%, alcanzando los 24.471 millones de euros el pasado año. Sin embargo, en el primer trimestre de 2015 también los datos revelan un cambio de tendencia en estas variables. Las exportaciones mantienen máximos históricos (alcanzaron los 60.972,8 millones de euros), con un crecimiento (enero-marzo 2015 con respecto a enero-marzo 2014) del 4,4\% gracias al excelente comportamiento del mes de marzo. En dicho mes se efectuaron ventas al exterior por un valor de 23.218,3 millones de euros, cifra que representa un máximo en la serie histórica. El cambio se ha producido en las importaciones, cuyo avance se desacelera. De enero a marzo de 2015 alcanzaron los 66.496,8 millones de euros, mostrando tan solo un crecimiento, con respecto al mismo periodo de 2014, del 2,5\%.

Por esto, el objetivo de este trabajo, es analizar las previsiones económicas de la economía española en este periodo de recuperación, analizando a su vez los sectores económicos clave para la economía a través de la metodología de matrices de contabilidad social.

El trabajo se estructurará en cinco epígrafes diferentes, dedicando el primero a la introducción, para seguir en el segundo con una contextualización del entorno internacional. En el tercer epígrafe, se analizará mediante la metodología de Matrices de Contabilidad Social los sectores productivos más importantes en España por su capacidad para influir y ser influidos por otros sectores, para en el cuarto establecer las proyecciones estimadas por Funcas y el departamento de economía de la Universidad Loyola Andalucía. Para finalizar, el quinto epígrafe recogerá las principales conclusiones del estudio llevado a cabo. 


\section{EL ENTORNO INTERNACIONAL}

El año 2014 terminó para el contexto económico internacional con una compleja combinación de efectos positivos y negativos. Como ya se ha comentado, España mostraba a finales del pasado año claros síntomas de recuperación en un entorno europeo preocupante por el bajo crecimiento y el riesgo de deflación, mientras la economía mundial se debatía entre los riesgos de un estancamiento en los países avanzados, que por otro lado, podrían beneficiarse de una fuerte caída de los precios del petróleo, activando así el consumo, que daría lugar a una aceleración del crecimiento.

En este momento, si algo caracteriza al patrón de crecimiento mundial es la complejidad de las fuerzas y dinámicas concurrentes: tendencias a medio y largo plazo, shocks a escala global y algunos factores que singularizan la casuística de ciertas regiones o de determinados países. En el contexto global, países desarrollados y emergentes están registrando un considerable deterioro en su economía debido al efecto contagio provocado por los problemas de la economía china, que dan lugar a la reducción de la demanda de materias primas y por consiguiente a la bajada de sus precios, todo esto unido a la falta de tensiones inflacionistas a corto plazo y manteniendo, en general, las previsiones de inflación a largo plazo en niveles inferiores a los objetivos de política monetaria. El crecimiento moderado que experimenta la economía mundial se justifica por el freno (temporal) que ha sufrido la actividad en Estados Unidos, la desaceleración del grueso de las economías emergentes (en especial los casos de China, Rusia y Brasil) y por otras circunstancias como son los efectos favorables de un petróleo, todavía barato, y por la orientación de las políticas de las principales autoridades monetarias, entre las que conviene destacar el programa de compra de deuda pública impulsado por el Banco Central Europeo (BCE).

El consumo privado y las exportaciones compensan la debilidad de la inversión. Por su parte, la relajación de las condiciones de financiación y la depreciación del euro, como resultado de la política monetaria expansiva del $\mathrm{BCE}$, junto con un comportamiento favorable del precio del petróleo, están siendo cruciales para que la recuperación se consolide en esta zona.

Los próximos movimientos de la Reserva Federal (Fed), en función de la interpretación que dicho organismo haga de lo ocurrido en el ámbito interno cobrarán especial protagonismo. El freno a la actividad se justifica por la menor inversión de capital en el sector de la energía, y por el acusado descenso de las exportaciones. Además, un duro invierno, un dólar que se ha apreciado más rápido de lo esperado, algunos conflictos laborales y el débil crecimiento global han sido factores que también han influido negativamente. Por el contrario, el consumo interno contribuyó de manera favorable al avance del Producto Interior Bruto (PIB). A pesar del carácter temporal de esta desaceleración, el menor ritmo de 
avance ha levantado algunas dudas sobre la solidez del crecimiento y el previsible impacto de la apreciación del dólar.

En la Tabla 1 se recoge la evolución del PIB real para un grupo de países representativos del entorno internacional.

Tabla 1. PIB real

(Porcentaje de variación respecto al año anterior)

\begin{tabular}{|l|c|c|c|c|c|c|c|}
\hline & $2010^{* *}$ & $2011^{* *}$ & $2012^{* *}$ & $2013^{* *}$ & 2014 & $2015 \mathrm{e}^{*}$ & $2016 \mathrm{e}^{*}$ \\
\hline $\begin{array}{l}\text { Unión Europea } \\
\text { (EU-28) }\end{array}$ & 2,05 & 1,80 & $-0,41$ & 0,12 & 1,40 & 1,85 & 1,95 \\
\hline Euro área & 2.03 & 1.62 & -0.81 & -0.46 & 0.88 & 1.45 & 1.65 \\
\hline Alemania & 3,94 & 3,66 & 0,58 & 0,21 & 1,61 & 1,62 & 1,67 \\
\hline Francia & 1,97 & 2,08 & 0,33 & 0,29 & 0,36 & 1,16 & 1,49 \\
\hline Gran Bretaña & 1,91 & 1,65 & 0,66 & 1,67 & 2,55 & 2,72 & 2,33 \\
\hline Italia & 1,71 & 0,59 & $-2,77$ & $-1,70$ & $-0,42$ & 0,49 & 1,10 \\
\hline Polonia & 3,70 & 4,80 & 1,80 & 1,70 & 3,30 & 3,50 & 3,50 \\
\hline Estados Unidos & 2,53 & 1,60 & 2,32 & 2,22 & 2,39 & 3,14 & 3,06 \\
\hline Canadá & 3,37 & 2,96 & 1,92 & 2,00 & 2,53 & 2,16 & 2,03 \\
\hline Japón & 4,65 & $-0,45$ & 1,75 & 1,61 & $-0,06$ & 1,04 & 1,17 \\
\hline Brasil & 7,57 & 3,92 & 1,76 & 2,74 & 0,15 & $-1,03$ & 0,98 \\
\hline Rusia & 4,50 & 4,30 & 3,40 & 1,30 & 0,62 & $-3,83$ & $-1,10$ \\
\hline India & 10,26 & 6,64 & 5,08 & 6,90 & 7,17 & 7,46 & 7,47 \\
\hline China & 10,41 & 9,30 & 7,76 & 7,75 & 7,36 & 6,76 & 6,30 \\
\hline Sudáfrica & 3,04 & 3,21 & 2,22 & 2,21 & 1,53 & 2,00 & 2,10 \\
\hline México & 5,11 & 4,05 & 4,01 & 1,39 & 2,13 & 3,00 & 3,32 \\
\hline Indonesia & 6,38 & 6,17 & 6,03 & 5,58 & 5,03 & 5,20 & 5,50 \\
\hline Nigeria & 10,60 & 4,90 & 4,30 & 5,40 & 6,30 & 4,80 & 5,00 \\
\hline Turquía & 9,16 & 8,77 & 2,13 & 4,12 & 2,90 & 3,15 & 3,60 \\
\hline Australia & 2,26 & 2,72 & 3,60 & 2,06 & 2,71 & 2,85 & 3,16 \\
\hline
\end{tabular}

$\mathrm{e}^{*}$ Dato estimado

** Excluida Letonia para el cálculo del dato de la Euro Área.

Fuente: elaboración propia a partir de datos del Fondo Monetario Internacional. 


\section{Alejandro Cardenete y M. Carmen Delgado}

Respecto a los países emergentes, su ritmo de crecimiento sigue siendo muy moderado, destacando los casos de las economías de China, Brasil o Rusia y las de algunos países exportadores de petróleo. No se debe obviar que, desde 2010, los mercados emergentes han experimentado una progresiva desaceleración. En China, el crecimiento económico se ha visto condicionado por la menor contribución de la inversión y también por algunas medidas de política económica cuyo objetivo ha sido limitar la fuerte expansión del crédito y el boom inmobiliario. Las tasas de crecimiento se desaceleran desde 2011 y, previsiblemente, lo seguirán haciendo en los próximos años debido a una disminución del potencial de fuerza de trabajo y una transición generalizada a una tasa de inversión algo menor, debiéndose considerar también las dificultades que conlleva pasar de un modelo de crecimiento orientado al exterior a otro basado en la demanda de consumo interna. Las perspectivas para Brasil se ven afectadas por la sequía, el endurecimiento de las políticas macroeconómicas y la falta de empuje del sector privado. En el caso de Rusia, las perspectivas de crecimiento evidencian el impacto económico de la fuerte caída de los precios del petróleo y el recrudecimiento de las tensiones geopolíticas. Por su parte, para otros mercados emergentes exportadores de materias primas, se espera que el impacto de la caída de los precios del petróleo y de otras materias primas, en los términos de intercambio y los ingresos reales, perjudique el crecimiento a medio plazo.

\section{ANÁLISIS SECTORIAL DE LA ECONOMÍA ESPAÑOLA}

La metodología de análisis multisectorial está basada en los modelos de Leontief, apoyados en las Matrices de Contabilidad Social (MCS). Las matrices de contabilidad social siguen la lógica de la tablas input - output, intentando corregir y superar algunas de las limitaciones de las bases de datos más convencionales usadas para el análisis económico. Por este motivo se puede decir que la MCS es una importante base de datos, organizada en forma de tabla de doble entrada, donde está recogida información económica y social de las transacciones habidas entre todos los agentes económicos. Éstas son bases de datos donde se recogen transacciones económicas, en términos de flujos de rentas, que permiten extraer información sobre los diferentes agentes económicos, tales como productores, consumidores, administración pública y sector exterior, así como sobre el comportamiento de los factores productivos.

El uso de las matrices de contabilidad social fue iniciado por Stone (1962) que publicaron una MCS para el Reino Unido. Sin embargo, dada su utilidad para conocer las relaciones intersectoriales de la economía y la distribución de la renta, las primeras MCS fueron elaboradas para países en vías de desarrollo con 
la finalidad de poner en marcha programas que supusieran una reducción de la pobreza en estos países. Entre otras, hay que destacar la MCS de Sri Lanka elaborada por Pyatt (1977) por el impulso que se dio en este campo y sus aplicaciones, con especial referencia al análisis de multiplicadores Pyatt y Round, (1979).

Una MCS recoge información económica y social relevante de todos los agentes económicos, manifestándose en todas aquellas transacciones realizadas entre los mismos durante un período de tiempo; transacciones que describen operaciones de producción, de distribución y utilización de la renta y de acumulación, tanto dentro de la propia economía como aquellas realizadas con el resto del mundo.

Una MCS amplía la información contenida en una tabla input-output, ya que, además de incluir a ésta, incluyen todos los flujos entre el valor añadido y la demanda final. Por tanto, en una MCS queda reflejado el flujo circular de la renta de una economía.

Si nos fijamos es la Figura 1 podemos ver que son las empresas las que producen los bienes y servicios utilizando los factores productivos suministrados por las economías domésticas. Para ello, las empresas pagan por el uso de estos factores, lo que constituyen las rentas de las economías domésticas. Estas rentas son utilizadas para la adquisición de bienes y servicios. Sin embargo, en una economía con sector público, éste detrae de los agentes económicos privados un flujo monetario (impuestos), retornado en forma de bienes públicos, transferencias y subvenciones (gasto público). Las relaciones con el sector exterior hacen que existan unos flujos monetarios de entrada (exportaciones) y salida (importaciones). La utilidad de una MCS se encuentra en que todos estos flujos monetarios quedan incorporados en ella.

Figura 1. Esquema simplificado del flujo circular de la renta

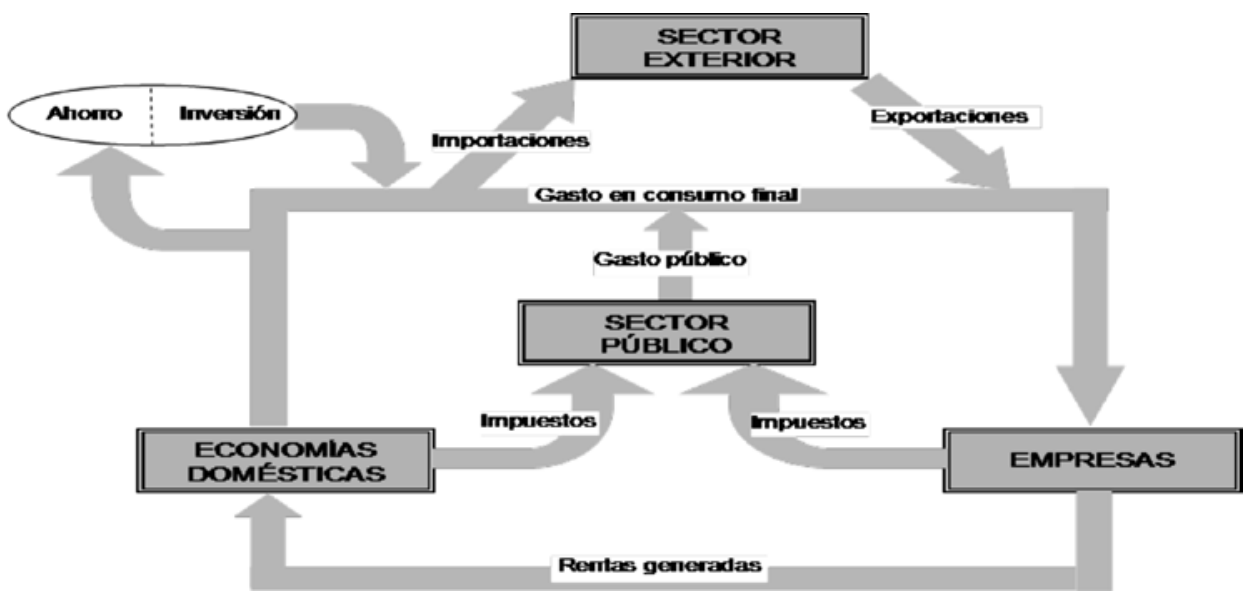

Fuente: Cardenete y Delgado (2011). 


\section{Alejandro Cardenete y M. Carmen Delgado}

Como en una MCS están representadas todas las transacciones realizadas por los agentes de la economía, se deben cumplir ciertas identidades contables, como que el gasto que realizan los agentes debe ser igual a la renta que han obtenido; esto es, la suma de cada columna debe ser igual a la suma de su correspondiente fila. La estructura de una MCS puede tomar diferentes formas dependiendo de las cuentas que la formen, existiendo, por tanto, una gran flexibilidad. A continuación se expone los bloques o submatrices de una matriz de contabilidad social:

\section{Tabla 2. Estructura simplificada de una matriz de contabilidad social}

\begin{tabular}{|c|c|c|c|c|c|}
\hline & Producción & $\begin{array}{c}\text { Factores } \\
\text { Productivos }\end{array}$ & $\begin{array}{c}\text { Sectores } \\
\text { Institucionales }\end{array}$ & Inversión & $\begin{array}{l}\text { Sector } \\
\text { Exterior }\end{array}$ \\
\hline Producción & $\begin{array}{l}\text { Consumos } \\
\text { Intermedios }\end{array}$ & & $\begin{array}{c}\text { Consumo } \\
\text { del Sector } \\
\text { Público y los } \\
\text { Hogares }\end{array}$ & $\begin{array}{l}\text { Formación } \\
\text { Bruta de } \\
\text { Capital }\end{array}$ & Exportaciones \\
\hline $\begin{array}{l}\text { Factores } \\
\text { Productivos }\end{array}$ & $\begin{array}{l}\text { Pagos de VA } \\
\text { a los factores }\end{array}$ & & & & \\
\hline $\begin{array}{l}\text { Sectores } \\
\text { Institucionales }\end{array}$ & $\begin{array}{c}\text { Impuestos s/ } \\
\text { actividades } \\
\text { y bienes y } \\
\text { servicios }\end{array}$ & $\begin{array}{l}\text { Asignación } \\
\text { de ingreso de } \\
\text { los factores a } \\
\text { los Sectores } \\
\text { Instit. }\end{array}$ & $\begin{array}{c}\text { Transferencias } \\
\text { corrientes } \\
\text { entre los } \\
\text { Sectores } \\
\text { Instit. }\end{array}$ & $\begin{array}{l}\text { Impuestos } \\
\text { s/ bienes de } \\
\text { capital }\end{array}$ & $\begin{array}{c}\text { Transferencias } \\
\text { del Resto del } \\
\text { Mundo }\end{array}$ \\
\hline Inversión & & $\begin{array}{l}\text { Consumo de } \\
\text { capital fijo }\end{array}$ & $\begin{array}{c}\text { Ahorro de los } \\
\text { sectores insti- } \\
\text { tucionales }\end{array}$ & & $\begin{array}{l}\text { Ahorro } \\
\text { exterior }\end{array}$ \\
\hline $\begin{array}{l}\text { Sector } \\
\text { Exterior }\end{array}$ & Importaciones & & $\begin{array}{l}\text { Transferen- } \\
\text { cias al Resto } \\
\text { del Mundo }\end{array}$ & & \\
\hline
\end{tabular}

Fuente: Cardenete y Moniche (2001).

- Matriz de consumos intermedios: en esta matriz se recogen las transacciones de bienes y servicios intermedios entre los sectores productivos y entre éstos y las Administraciones Públicas. La suma de las entradas de cada columna indica las compras de bienes intermedios realizadas por cada sector y la suma de la filas representan las ventas efectuadas por cada uno de los sectores. La suma de todas las entradas da como resultado la agregación del consumo intermedio de la economía.

- Matriz de valor añadido: recoge la descomposición de los recursos utilizados por cada sector productivo. Las partidas incluidas son las siguientes: trabajo, 
formado por los sueldos y salarios brutos; capital, formado por el consumo de capital fijo, el excedente neto de explotación y las rentas mixtas; cotizaciones a la Seguridad Social por parte de los empleadores.

- Matriz de demanda final: situada en la parte superior derecha de la Tabla input - output, está formada por las columnas de consumidores, que representa el gasto total de las economías domésticas, administración pública, que recoge el consumo del sector público como agente económico; ahorro/ inversión, formada por la cuenta exterior, donde ahora se reflejan las exportaciones a la Unión Europea y al resto del mundo.

Estas tres matrices resumen las transacciones entre los agentes de la economía y deben cumplir la identidad contable de que la producción bruta total es igual a la demanda total.

- Matriz de cierre: esta matriz es la que permite el cierre del flujo circular de la renta de la estructura económica multisectorial que representa una matriz de contabilidad social. Se encuentra en la parte inferior derecha de la MCS y se trata de la parte que realmente completa el marco contable de las Tablas Input-Output (TIO). Las tablas de origen y destino describen los procesos de producción y utilización de los bienes y servicios, pero no incorporan las interrelaciones entre el valor añadido y el gasto final. Estas interrelaciones sí aparecen reflejadas en la MCS, lo que permite mostrar en su totalidad el flujo circular de la renta. En las filas y columnas correspondientes a hogares y Sector Público aparecen por filas los recursos totales para hacer frente a sus gastos en consumo e inversión. Por columnas se muestran los empleos en gastos finales, pago de impuestos o ahorro.

En la metodología que se aplica en este apartado, exactamente se extiende el Modelo de Leontief a Modelos MCS lineales, para evaluar, mediante el cálculo de los multiplicadores, como son los cambios en producción, renta o empleo en las cuentas endógenas a causa de políticas que modifiquen las cuentas exógenas para las ramas productivas de la economía española. La Teoría de multiplicadores fue iniciada por Stone (1978), y Pyatt y Round (1979), desarrollándose posteriormente con trabajos como los de Defourney y Thorbecke (1984). Estos métodos se basan en obtener información, a partir de las matrices inversas derivadas de los modelos de Leontief (1941) y de Ghosh (1958) aplicados a la MCS, sobre la capacidad que tiene un sector de expandir aumentos de demanda o de costes, respectivamente. La metodología se apoya en la combinación de dos tipos de enlaces intersectoriales, los Backward linkages (BL), o vínculos hacia atrás, y los Forward linkages (FL), o vínculos hacia delante. El BL aporta información sobre el efecto que tiene el aumento de la demanda de un sector en la economía, es 


\section{Alejandro Cardenete y M. Carmen Delgado}

decir, de donde proceden los inputs que un sector requiere para incrementar su producción. Los FL ofrecen información sobre el efecto en el resto de sectores de cambios en el valor de los inputs primarios, y, por tanto, en la producción, de un sector concreto, es decir, cual es el destino de la producción de un sector y en qué medida afecta al resto la variación en su valoración. En este apartado se desarrolla esta metodología, que se aplicará a la MCS de la economía española, con el objetivo de identificar los sectores clave y las principales interrelaciones del país.

Una vez que los enlaces son calculados, su interpretación es como sigue: aquellos sectores con FL superior a uno son sectores con capacidad de dispersión de costes, o lo que es lo mismo, los cambios en la cuantía de su valor añadido afectan más al sistema que la media. Los sectores con BL superior a uno tienen poder de dispersión, es decir, una variación en su producción influye más en el sistema que la media. Ambos índices nos permiten, además, identificar cuáles son los sectores clave de una economía, que se definen como aquellos sectores que tienen tanto poder de dispersión de variaciones en la demanda (BL.j>1), cómo capacidad de dispersión de variaciones en los costes (FL.j>1). En la tabla 3 se puede observar una clasificación sectorial en función del peso de los BL y los FL.

Tabla 3. Clasificación sectorial a partir de los BL y FL

\begin{tabular}{|c|c|c|}
\cline { 2 - 3 } \multicolumn{1}{c|}{} & BL $<$ Promedio $(\mathrm{BL})$ & BL $>$ Promedio $(\mathrm{BL})$ \\
\hline $\mathrm{FL}<$ Promedio $(\mathrm{FL})$ & Sectores independientes & Sectores impulsores \\
\hline $\mathrm{FL}>$ Promedio $(\mathrm{FL})$ & Sectores base & Sectores clave \\
\hline
\end{tabular}

Fuente: Cardenete y Delgado (2011).

- Sectores clave: demanda y ofrecen grandes cantidades de inputs intermedios que suelen estar muy repartidas entre las distintas ramas, son una parte muy importante del flujo de toda la economía, al ser fuertemente impulsados por variaciones en cualquier sector, y este impulso afecta a su vez de forma importante al resto del sistema con lo que pueden provocar a un aumento generalizado de la actividad económica.

- Sectores base o estratégicos: son aquellos que presentan una baja demanda de inputs, siendo el destino de su producción preferentemente el uso intermedio, es decir, sirve de input a otros sectores, por lo que sus variaciones, en precios o cantidades, afectan de forma importante al resto.

- Sectores impulsores: son grandes demandantes de inputs intermedios y dada la capacidad que tienen de inducir otras actividades pueden afectar en mayor cuantía al crecimiento global de la economía. 
- Sectores independientes: son aquellos que presentan unos encadenamientos hacia delante y hacia atrás por debajo de la media, por lo que afectan y son afectados por el sistema de forma inferior a la media.

A continuación, se puede ver la clasificación de sectores en los grupos arriba señalados para el país en cuestión:

\section{Figura 2. Clasificación sectorial España}

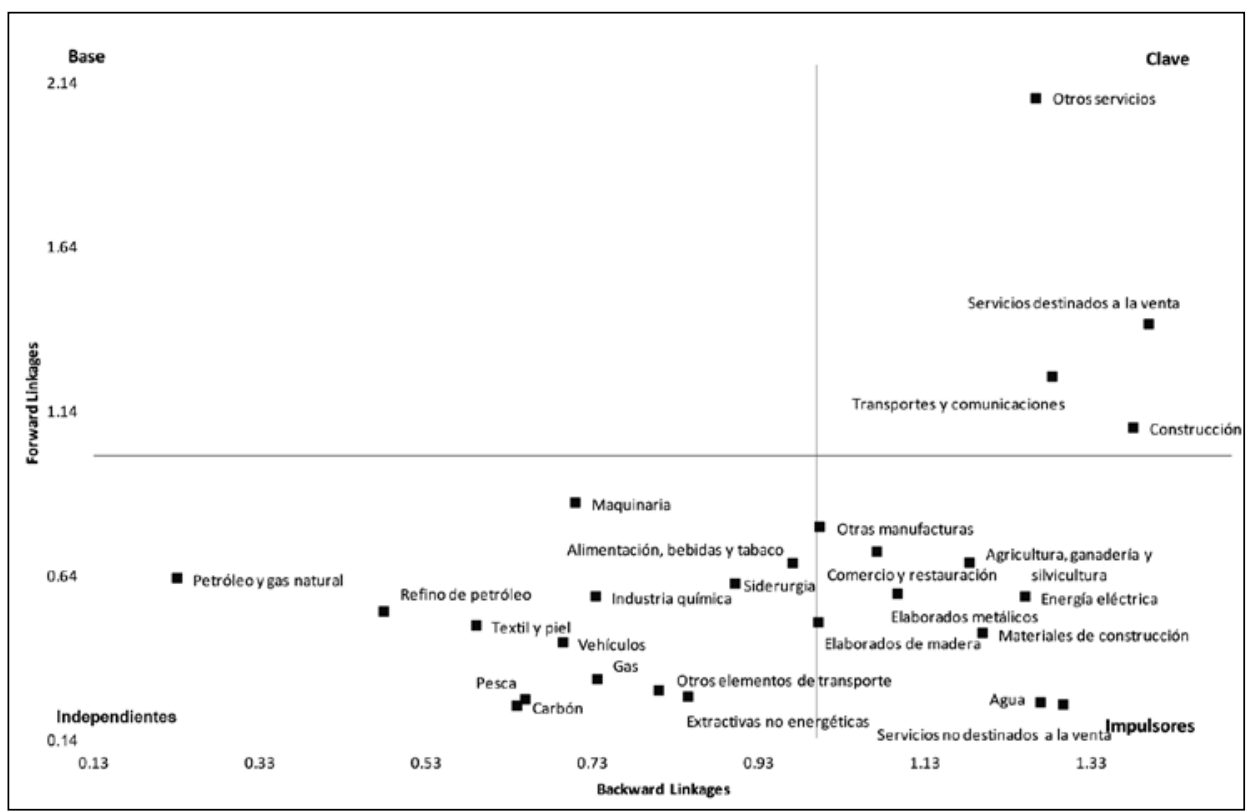

Fuente: elaboración propia.

En la Figura 2 se puede observar como de los sectores recogidos en la matriz de contabilidad social para España, son sectores clave las ramas de Construcción, Transportes y comunicaciones, Otros servicios ${ }^{3}$ y Servicios destinados a la venta ${ }^{4}$.

\footnotetext{
3 "Otros servicios" incluye: Programación, consultoría y otras actividades relacionadas con la informática; Actividades auxiliares a los servicios financieros y a los seguros; recogida, tratamiento y eliminación de residuos; publicidad y estudios de mercado; Actividades de seguridad e investigación; y actividades jurídicas y de contabilidad.

4 "Servicios destinados a la venta" incluye: Ingeniería civil, artes gráficas y reproducción de soportes grabados; Actividades de sedes centrales; Servicio técnico de arquitectura e ingeniería; Actividades veterinarias; Actividades de alquiler; y otros servicios personales.
} 


\section{Alejandro Cardenete y M. Carmen Delgado}

Los resultados muestran el fuerte peso que el sector servicios y construcción continúan teniendo en la economía española, manteniéndose su protagonismo en la estructura sectorial. Al igual ocurre con la importancia del sector comercio y agricultura, que aunque no se clasifican como sector clave, sí podríamos decir que son potencialmente clave, manteniendo su participación en las magnitudes económicas y su importancia relativa en el conjunto nacional. Esto confirma la tendencia al estancamiento de la estructura económica española, ya que no se ha producido cambio estructural alguno, manteniéndose de esta forma los mismos vínculos entre sectores en cuanto a motores de crecimiento de la economía se refiere.

\section{PROYECCIONES PARA ESPAÑA}

De acuerdo con los indicadores disponibles relativos al segundo trimestre de 2015 se puede hablar de una aceleración del crecimiento económico en dicho periodo. Debido a esto la previsión de crecimiento del PIB para el cierre del 2015 se ha revisado al alza y se sitúa según Funcas en el 3,3 \%. Según Laborda y Fernández (2015) en su informe para Funcas explican que la economía está siendo impulsada tanto por factores endógenos cíclicos y mejoras estructurales, como por el impacto de un conjunto de shocks externos de carácter transitorio, entre los que destaca la caída del precio del petróleo, el pago a los empleados públicos de parte de la paga extraordinaria que fue eliminada en diciembre de 2012, la rebaja del impuesto sobre la renta, la fuerte caída de los tipos de interés y la relajación del gasto público como consecuencia del ciclo electoral. A partir del tercer trimestre se espera el agotamiento progresivo del impacto expansivo de algunos de dichos shocks, por lo que se espera una desaceleración del ritmo de crecimiento. Por el mismo motivo, los autores comentan que el crecimiento previsto para 2016 se sitúa en el 3,0\%, es decir, por debajo del esperado para 2015, aunque dos décimas porcentuales por encima de lo que estimaron en anteriores previsiones. Según comentan, en ambos años el crecimiento del PIB provendrá enteramente de la aportación de la demanda interna, ya que la del sector exterior será negativa del orden de medio punto porcentual. Desde que se inició la recuperación, en el tercer trimestre de 2013, la economía ha crecido un 3,4\%, lo que significa que se ha recuperado el 39\% del PIB real que se había destruido durante la recesión, y, conforme al escenario actual de previsiones, al final de 2016 se habrá recuperado la totalidad del mismo. A continuación, en la Figura 3 elaborada en este informe de previsiones se puede ver reflejado lo comentado anteriormente: 


\section{Figura 3. PIB, demanda nacional y saldo exterior}

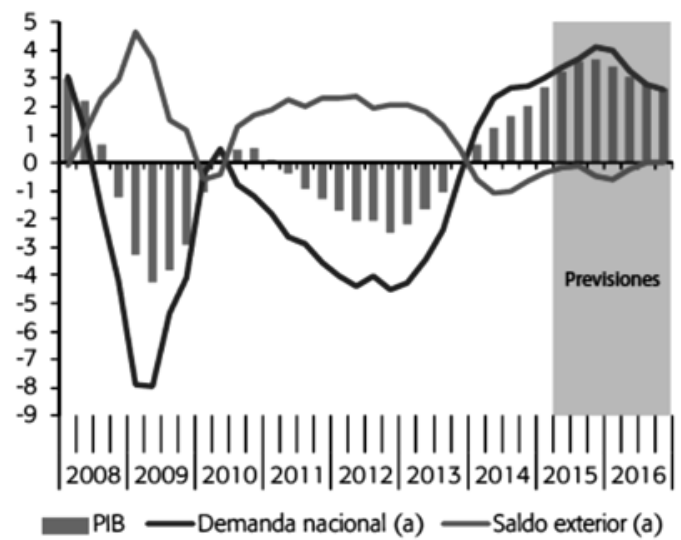

(a) Aportación al crecimiento del PIB en puntos porcentuales.

Fuente: Laborda y Fernández (2015).

En este mismo informe, también son analizadas variables como el empleo, en términos de puestos de trabajo equivalentes a tiempo completo, y según Laborda y Fernández (2015) aumentará un 3\% en 2015 y un 2,5\% en 2016, en ambos casos más de lo estimado en las anteriores previsiones. Esto supone la creación de más de 900.000 empleos a lo largo de los dos ejercicios, como puede verse en la Figura 4.

\section{Figura 4. Empleo y paro}

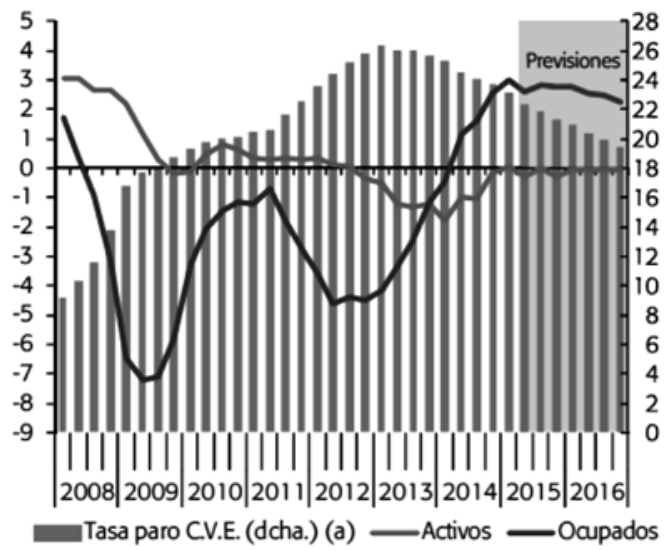

(a) Porcentaje de la poblaciónen edad de trabajar.

Fuente: Laborda y Fernández (2015). 


\section{Alejandro Cardenete y M. Carmen Delgado}

Los autores apuntan que esto significa que, si hasta el primer trimestre de 2015 se había recuperado el $13 \%$ del empleo que se había perdido durante la crisis, al final de 2016 se habrá recuperado la tercera parte del mismo. La tasa de paro media anual descenderá 2,2 puntos porcentuales de la población activa en 2015 y otros 2 puntos en 2016, hasta el 22,2\% y 20,2\% respectivamente. Los costes laborales unitarios aumentarán ligeramente en los dos ejercicios, aunque sin agotar el crecimiento de la productividad.

Por otra parte hablan de la ausencia de presiones por parte de los costes y de la demanda mantendrá la inflación generada por la economía española en cifras moderadas, esperándose un aumento del deflactor del PIB inferior al $1 \%$ tanto en 2015 como en 2016. Debido a los precios de las importaciones, la inflación de los precios de consumo se situará notablemente por debajo de esta cifra en este año $(-0,2 \%)$ y algo por encima en el próximo, según se puede observar en la Figura 5.

\section{Figura 5. Inflación}

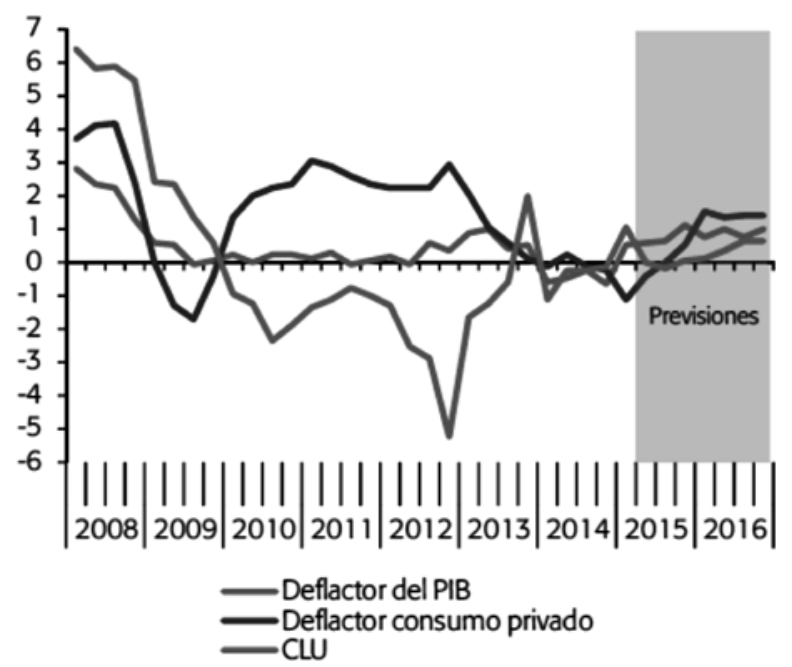

Fuente: Laborda y Fernández (2015).

Para finalizar esta sección, a continuación presentamos otra óptica de previsiones económicas, realizada en el mes de junio por el departamento de economía de la Universidad Loyola Andalucía, con la metodología de análisis univariante de Box-Jenkins vía los modelos ARIMA (Modelos Autorregresivos Integrados de Media Móvil), en las que se presentan proyecciones de las variables macroeconómicas PIB, Índice de Precios al Consumo (IPC) y desempleo. 
Tabla 4. Proyecciones económicas para España (PIB, Desempleo, IPC).

\begin{tabular}{|l|c|c|c|}
\cline { 2 - 3 } \multicolumn{1}{c|}{} & $2015 \mathrm{~T} 2$ & $2015 \mathrm{~T} 3$ & \multirow{2}{*}{2015} \\
\cline { 2 - 3 } \multicolumn{1}{c|}{} & Intertrimestral & Intertrimestral & 3,1 \\
\hline PIB (\% variación) & 0,9 & 1,3 & \multicolumn{1}{c|}{} \\
\hline $\begin{array}{l}\text { Desempleo } \\
\text { (\% variación) }\end{array}$ & $-5,5$ & $-4,0$ & $21,0-22,0$ \\
\hline $\begin{array}{l}\text { Tasa de paro } \\
\text { (\% población activa) }\end{array}$ & 22,5 & 21,6 & $0,0-0,5$ \\
\hline IPC (\% variación) & 0,3 & 0,6 & \\
\hline
\end{tabular}

Fuente: Cardenete et al. (2015).

Como se puede observar, en la Tabla 4 se presentan las previsiones para el segundo y tercer trimestre (T2 y T3 respectivamente) del año 2015, así como la previsión del cierre del año. De acuerdo con las estimaciones que se realizaron en el número de primavera del Loyola Economic Outlook y debido a la mejora en la coyuntura económica producida, la previsión del crecimiento anual del PIB para el año 2015 ha sido revisada al alza, alcanzando un 3,1\%. Así, se estima también que el crecimiento del PIB en el T2 será del 0,9\% y en el T3 del 1,3\%. En cuanto a la evolución de la tasa de desempleo, en ambos trimestres se proyecta una disminución, que será del 5,5\% para el T2 y del 4,0\% para el T3, situándose la tasa de paro en el 22,5\% y 21,6\% respectivamente. En cuanto al IPC la previsión apunta a una ligera subida en el T2 y el T3 de 0,3\% y 0,6\% respectivamente.

\section{CONCLUSIONES}

En este trabajo se realiza un análisis de las previsiones económicas de la economía española en este periodo de recuperación, detectando a su vez los sectores económicos clave para la economía española a través de la metodología de matrices de contabilidad social.

La aplicación de un modelo MCS lineal a través del análisis de multiplicadores ha permitido clasificar los sectores productivos españoles según su capacidad para influir y ser influidos por cambios en ellos mismos y en el resto del sistema, considerando unos valores medios de referencia. Así pues, se han identificado como sectores clave las ramas de Construcción, Transportes y comunicaciones, Otros servicios y Servicios destinados a la venta, que aunque los efectos de la crisis se han hecho notar a través de una reducción en su papel como impulsores de crecimiento y empleo del país, aún siguen clasificándose como los sectores más 


\section{Alejandro Cardenete y M. Carmen Delgado}

importantes. Este análisis pone de relieve la estabilidad de la estructura económica española en cuanto a sectores dinamizadores de la economía se refiere, así como la dependencia del sector construcción, apuntada anteriormente.

Por otra parte y cumpliendo otro de los objetivos del trabajo, se ha realizado un análisis de las previsiones económicas suministradas por dos informes diferentes: el primero de ellos, correspondiente al servicio de estudio de Funcas, pone de manifiesto un crecimiento del PIB para el año 2015 de 3,3\%, un aumento del empleo del $3 \%$, un descenso de la tasa de paro media anual de 2,2 puntos porcentuales de la población activa, alcanzando la tasa de desempleo el 22,2\%; por último la inflación generada se situará en este año en el -0,2\%. El segundo de ellos, realizado por el departamento de economía de la Universidad Loyola Andalucía, sitúa el crecimiento del PIB anual en el 3,1\%, la tasa de paro en un abanico de 21 al $22 \%$ y el índice de precios al consumo en una variación que oscilará entre 0 y $0,5 \%$.

Sin duda alguna, el entorno nacional, y por tanto estas previsiones apuntadas, se verán sujetas a cambios provocados por el entorno internacional que afectará en lo que resta del año y en 2016 a las exportaciones españolas y a la inversión. Como ya se ha comentado anteriormente, junto a los efectos negativos que se están produciendo y que se pueden producir, también existen otros que se podrían catalogar como efectos positivos, que pueden compensar casi en su totalidad a los efectos llamados negativos. España, sin lugar a dudas, y tal y como apunta el estudio analizado de Laborda y Fernández (2015), se verá beneficiada de los bajos precios del petróleo y de las materias primas así como de mejores condiciones financieras, ya que al repliegue de los inversores a las zonas más seguras, se unirá probablemente una política monetaria más relajada por parte de los bancos centrales de los países desarrollados. Aunque, en cuanto a las condiciones financieras, el riesgo para España es que los inversores pidan más prima de riesgo si el clima político se deteriora tras las elecciones catalanas y generales. También esto podría afectar al clima de confianza y a las expectativas y, como consecuencia, al consumo y sobre todo a la inversión.

\section{BIBLIOGRAFÍA}

CARDENETE, M. A. y DELGADO, M. C. (2011), "Análisis de la estructura de la economía georgiana", Papeles de Europa, núm. 23, pp. 21-42.

CARDENETE, M. A. y MONICHE, L. (2001), "El nuevo marco Input-Output y la SAM de Andalucía para 1995", Cuadernos de Ciencias Económicas y Empresariales, núm. 41, pp. 13-31.

CARDENETE, M. A., NEKHAY, O., AMADOR, L., DELGADO, M. C., MOLERO, S. y SALAZAR-ORDÓÑEZ, M. (2015), Loyola Economic 
Outlook. Proyecciones Macroeconómicas (junio 2015). Universidad Loyola, Sevilla.

DEFOURNEY, J. y THORBEKE, E. (1984), "Structural Path Analysis and Multiplier Decomposition within a Social Accounting Matrix framework", The Economic Journal, núm. 94 (373), pp. 111-136.

GHOSH, A. (1958), "Input-Output Approach in Allocation System", Economica, núm. 25, pp. 58-64.

LABORDA, A. y FERNÁNDEZ M. J. (2015), "El crecimiento de la economía española supera las expectativas", Cuadernos de Información Económica, núm. 247 Economía y Finanzas Española (julio-agosto 2015).

LEONTIEF, W. (1941), The Structure of American Economy, 1919-1924: An Empirical Application of Equilibrium Analysis, Harvard University Press, Cambridge.

PYATT, G. (1977), Social Accounting for Development Planning with Special Reference to Sri Lanka, Cambridge, Cambridge University Press.

PYATT, G. y ROUND, J. I. (1979), "Accounting and fixed price multipliers in a Social Accounting Matrix framework", The Economic Journal, núm. 89, pp. 850-873.

STONE, R. (1962), A Social Accounting Matrix for 1960 en A Programme for Growth, Chapman and Hall Ltd. (Eds.), Cambridge.

STONE, R. (1978), The Disaggregation of the Household Sector in the National Accounts, Paper presentado en World Bank Conference on Social Accounting Methods in Development Planning, Cambridge, Reino Unido, 16-21 de abril de 1978. 
